\title{
Letter from Henryk Iwaniec to S.-T. Yau Regarding the Work of Yitang Zhang
}

On May 24, 2013, Shing-Tung Yau (Harvard University) received the following letter from Henryk Iwaniec (Rutgers University), one of the world's leading experts on analytic number theory:

\section{Dear Yau,}

I was pleased to discuss this fascinating story with you by telephone a few days ago. As promised I am sending to you my impressions.

The result of Zhang has already been praised by the press and on internet blogs so highly that there would be no value added, if I repeat similar words of admiration. Thus I am going to relate impressions from the perspective of someone who read and checked the paper. I hope this will tell something about Zhang as well as about the subject matter.

Yitang Zhang was not well-known to specialists in number theory before his fantastic paper on prime numbers was recognized by the Annals of Mathematics three weeks ago. But he possessed the knowledge of the most sophisticated areas of analytic number theory, and he could use it all with ease. Also, he was able to make a breakthrough where many investigators were stuck, not because something little was overlooked, but because of new, clever arrangements which he introduced and brilliantly executed. You could sense immediately that the work had a great chance to be fine by looking at the clear and logical architecture of the arguments. It does not mean the paper is simple or elementary. To the contrary, the work of Zhang constitutes the state-of-the art of analytic number theory. It also borrows gracefully from other areas, for example, it makes use indirectly of the Riemann hypothesis for varieties over finite field. Zhang's work will trigger a lasting avalanche of refinements and improvements with many innovations. Overnight Zhang redirected the focus of analytic number theory. How long do we need to wait to see what comes next?

Be in touch.

Best,

Henryk 